\section{Zwischen Flächenreserve und Nahversorgung}

\section{Auf Landwirtschaft im Umland von Großstädten wirken unterschiedliche Inter- essen und Ansprüche ein: Sicherung von Flächenpotenzial für Siedlungszwecke, Erhalt von Freiraum und Kulturlandschaft für Naherholung sowie Aufgaben des Landschafts- und Wasserschutzes. Gleichzeitig haben landwirtschaftliche $\mathrm{Be}$ - triebe eine Versorgungsfunktion für die städtische Bevölkerung und bieten Dienstleistungen an. Diese vielfältigen Beziehungen bieten Potenziale für eine nachhaltige Gestaltung, die bisher noch wenig genutzt werden.}

$\mathrm{I}$ Von Thomas Fiebig n einigen Großstädten hat die Wahrnehmung dieser Zusammenhänge inzwischen zu Initiativen im Agrarbereich geführt. Handlungsschwerpunkte sind dabei die Verbesserung der Koordinierung von Ansprüchen an die landwirtschaftliche Fläche, der Aufbau kleinräumiger Wirtschaftskreisläufe im Nahrungsmittelbereich und die Forcierung einer umweltschonenderen Ausrichtung der Produktion (1).

Großstadt bedeutet für die landwirtschaftlichen Betriebe insbesondere die Nähe zu einem hohen Nachfragepotenzial für die eigenen Produkte. So stehen zum Beispiel in Saarbrücken den 360.000 Einwohnern 180 Höfe gegenüber, in Wuppertal sind es bei der gleichen Einwohnerzahl rund 90 aktive Landwirte, davon die Hälfte mit Direktvermarktung von landwirtschaftlichen Produkten (2). Für die unterschiedlichen Produkte sind verschiedene Absatzwege entstanden, wie die Direktvermarktung, Frei-Haus-Lieferung, Marktbeschickung oder Belieferung von Großverbrauchern. Von Seiten der Verbraucher besteht ein hoher Anspruch an Qualität, Herkunft und Verfügbarkeit von Nahrungsmitteln. Die Ergebnisse und Erfahrungen aus den verschiedenen Initiativen zeigen die ökonomischen Chancen auf, die im Umfang der Nachfrage nach Lebensmitteln, der höheren Transparenz der Herkunft und den kleinräumigeren Transportbeziehungen liegen. Durch die Ergänzung der Lebensmittelvermarktung mit Dienstleistungen und Freizeitangeboten können zusätzliche Einkommensmöglichkeiten geschaffen werden.

In diesem Zusammenhang gilt aber auch:

- Die Direktvermarktung von Lebensmitteln konkurriert bei den Verbrauchern mit dem Angebot des Lebensmittelgroß- und -einzelhandels, das preisgünstig, saisonal unabhängig und in ausreichender Menge vorhanden ist.

- Dienstleistungen zur Einkommensergänzung (unter anderem Landschafts-/ Gartenpflege und Pferdehaltung) treffen in vielen Bereichen auf bestehende Anbieter, so das häufig Überangebote entstehen.

Zweites zentrales Handlungsfeld der Initiativen von Großstädten im Agrarbereich ist der Umgang mit der landwirtschaftlichen Produktionsfläche. Neben die Frage, welche Flächen zukünftig für die Siedlungsentwicklung benötigt werden, treten Naherholungsbedürfnisse der Stadtbewohner und Ansprüche des Landschafts- und Freiraumschutzes. Die Planung löst die dabei entstehenden Konflikte in der Regel zugunsten der in ihrer Bedeutung für die Stadt ,wichtigeren“ Nutzungen. Verkaufserlöse für Bauland oder Leistungen für Pflegemaßnahmen auf Ausgleichsflächen bieten in diesem Zusammenhang nur einen unvollständigen Interessensausgleich. In diesem Konfliktfeld ist die Organisation von Entscheidungsprozessen notwendig, die bewusst alle Ansprïche ausreichend berïcksichtigen.

\section{Nachhaltige Beziehung?}

Eine nachhaltige Gestaltung der Beziehung zwischen (Groß-)Stadt und landwirtschaftlichen Betrieben in ihrem räumlichen Umfeld reduziert sich nicht allein auf die Flächeninanspruchnahme für Siedlungszwecke. Der in der nachhaltigen Stadtentwicklung propagierte Leitsatz „Innen- vor Außenentwicklung“ kann nur eingeschränkt diesen Konflikt mindern. Die Strategie greift lediglich in Städten mit einem Innenentwicklungspotenzial. Die in den Diskussionen nachhaltiger Stadtentwicklung entwickelten Ziele sind stark auf die
Optimierung städtischer Funktionen bezogen. Die Landwirtschaft findet in diesem Kontext nur am Rande Beachtung (3).

Ein Ansatzpunkt zur Konfliktlösung ist die Etablierung eines kontinuierlichen Kommunikationsprozesses zwischen Landwirtschaft und Verwaltung über die Themenbereiche Flächennutzung und Landschafts-/ Naturschutzplanung. Hier kann Konflikten und gegenseitigen Blockaden im Vorfeld von Planungen begegnet werden. Dafür sind geeignete Arbeits- und Organisationsformen aufzubauen oder bereits laufende Prozesse wie die Lokale Agenda 21 zu nutzen.

In der Gestaltung der ökonomischen Austauschprozesse haben die verschiedenen Initiativen bisher gezeigt, dass es darum geht, die örtliche Landwirtschaft und ihre Produkte stärker bekannt zu machen. Dies kann über eine Intensivierung der Öffentlichkeitsarbeit und Vermarktungsaktivitäten, aber auch durch eine Integration der Landwirtschaft in Umweltbildungsaktivitäten geschehen.

In den Vermarktungswegen für landwirtschaftliche Produkte muss eine stärkere Zusammenarbeit zwischen Betrieben mit dem Ziel der Ergänzung ihrer Produktpalette erreicht werden. Netzwerke von Landwirten, Verarbeitern und Vermarktern können eine Belebung oder einen Neuaufbau von Produktlinien und Wirtschaftskreisläufen bewirken. So kann die Landwirtschaft im Umland von Großstädten eine ,nachhaltige“ Bedeutung erlangen, wie sie sich in der zunehmenden Akzeptanz von Wochenmärkten schon heute ausdrückt.

\section{Anmerkungen}

(1) Beispiele sind kommunale Agrar-Initiativen in Hannover und Münster. Vgl. A. Brink in: Agrarbündnis (Hrsg.): Landwirtschaft '94. Der kritische Agrarbericht, S. 52-56; Stadt Münster (Hrsg.): Umweltbericht 1998.

(2) Vgl. Kühlbach, K./ Thös, J. in: „Regionalvermarktung“, KTBL-Arbeitspapier 224, S. 60-83 sowie Molitor, R./ Nischwitz, G.: Landwirtschaft in Großstadtnähe. IÖW-Schriftenreihe 148/00. Berlin 2000 (im Erscheinen).

(3) Vgl. ILS NRW (Hrsg.): Nachhaltige Stadtentwicklung. Praxisbeispiele. Dortmund 1999, S. 23ff.

\section{Der Autor}

Thomas Fiebig ist freiberuflich in der Regional- und Stadtplanung tätig. Er ist in mehreren Projekten nachhaltiger Stadt- und Regionalentwicklung mit dem Montoring und der Prozessbeobachtung betraut. Kontakt: Hafenstraße 13, 48153 Münster, Tel. 0251/ 525369, Fax 0251/ 5395724 E-Mail: t-fiebig@muenster.de 
(c) 20I0 Authors; licensee IÖW and oekom verlag. This is an article distributed under the terms of the Creative Commons Attribution Non-Commercial No Derivates License (http://creativecommons.org/licenses/by-nc-nd/3.o/), which permits unrestricted use, distribution, and reproduction in any medium, provided the original work is properly cited. 